\title{
Systematic review and meta-analysis of cochlear implantation in deaf patients with large vestibular aqueduct deformity
}

\author{
Liping Pan ${ }^{1}$, Hongyan $\mathrm{Lin}^{1}, \mathrm{Xiao} \mathrm{Li}^{2}$, Shuai Liu ${ }^{3}$ \\ ${ }^{1}$ Department of Otolaryngology, Anyang Sixth People's Hospital, Anyang, China; ${ }^{2}$ Department of Otolaryngology, The First Affiliated Hospital of \\ Xinxiang Medical College, Xinxiang, China; ${ }^{3}$ Department of Otolaryngology, Anyang Hospital of Traditional Chinese Medicine, Anyang, China \\ Contributions: (I) Conception and design: L Pan, S Liu; (II) Administrative support: H Lin; (III) Provision of study materials or patients: L Pan, H \\ Lin, X Li; (IV) Collection and assembly of data: All authors; (V) Data analysis and interpretation: L Pan, X Li, S Liu; (VI) Manuscript writing: All \\ authors; (VII) Final approval of manuscript: All authors. \\ Correspondence to: Shuai Liu. Department of Otolaryngology, Anyang Hospital of Traditional Chinese Medicine, No. 150, Hongqi Road, Anyang, \\ China. Email: LSDXDX13837228006@163.com.
}

Background Abnormal inner ear anatomy increases the risk of cochlear implantation because only a certain number of neurons can input signals to the auditory cortex. Therefore, the effectiveness and safety of cochlear implantation for patients with large vestibular aqueduct deformity (LVAD) are controversial and we explored whether cochlear implantation can improve the hearing of patients with this defect.

Methods: Randomized controlled trials of cochlear implantation for the treatment of LVAD were retrieved from the CNKI, Baidu Academic, VIP Medical, Wanfang Data, PubMed, EBSCO, Medline, and Cochrane databases from the date of establishment of the database to July 20, 2021. Chinese and English search keywords included Large vestibular canal malformation, Deafness, large vestibular aqueduct, and Cochlear implants. We used RevMan 5.3 to evaluate the quality of the literature.

Results: A total of 5 documents that met the inclusion criteria were tested for overall heterogeneity. For the heterogeneity test of categories of auditory performance, Chi-squared $\left(\mathrm{Chi}^{2}\right)=4.00$, degrees of freedom (df) $=1, \mathrm{I}^{2}=75 \%>50 \%$, and $\mathrm{P}=0.05$. Overall analysis using a random-effects model showed no statistically considerable difference between the deformity group and the control group $[\mathrm{Z}=0.78$, mean difference $(M D)=-0.65,95 \%$ confidence interval (CI): -2.29 to $0.98, \mathrm{P}=0.43$ ]. There was no substantial difference in postoperative hearing ability between LVAD patients and those with normal inner ear structure. Continuous variables were used to describe the speech intelligibility rate (SIR) in a total of 48 cases, including 24 cases in the deformity group and 24 cases in the control group. For the heterogeneity test of the whole population, $\mathrm{Chi}^{2}=1.75$, df $=1, \mathrm{I}^{2}=43 \%<50 \%$, and $\mathrm{P}=0.19$. Overall analysis using a fixed-effects model showed that the difference between the deformity group and the control group was statistically considerable $(\mathrm{Z}=3.09, \mathrm{MD}$ $=-1.03,95 \% \mathrm{CI}:-1.69$ to $-0.38, \mathrm{P}=0.002)$.

Discussion: The meta-analysis results confirmed that with postoperative rehabilitation for LVAD patients with cochlear implants the clinical efficacy is similar to that of deaf patients with normal inner ear structure, providing an important basis for hearing restoration.

Keywords: Cochlear implantation; deafness; large vestibular aqueduct deformity (LVAD); meta-analysis

Submitted Oct 27, 2021. Accepted for publication Dec 06, 2021.

doi: 10.21037/apm-21-3327

View this article at: https://dx.doi.org/10.21037/apm-21-3327 


\section{Introduction}

Large vestibular aqueduct deformity (LVAD) is a congenital malformation of the inner ear, an autosomal recessive genetic disease, mainly due to enlargement of vestibular aqueduct, which belongs to congenital sensorineural deafness (1-3). Originally discovered and named by Harker et al. in 1999, it is essentially abnormal communication between the cerebrospinal fluid and perilymph (4). The incidence of LVAS was $1.5 \%$, accounting for $31.5 \%$ of congenital inner ear malformation. The vestibular aqueduct (VA) is a bony duct that contains a variety of components and tissues, including endolymphatic vessels, endolymphatic sacs, and venules. In addition, it also contains some connective tissue, perilymph, and dura mater (5). The VA starts from the medial vestibular wall., located between the vestibule and the endolymphatic sac, and extends backward and outward to open on the surface of the petrous cone of the temporal bone. The return veins of the semicircular canal and the utricle both enter the sigmoid sinus after passing through the VA.

The emergence of a large vestibular aqueduct (LVA) may be caused by cessation or disorder of the endolymphatic vessels during embryonic development (6). In the embryo, the VA is short, straight, and broad, and gradually becomes long and narrow with maturity, showing the typical inverted "J" shape $(7,8)$. Adverse factors affecting the early embryonic development of the inner ear may cause the VA to remain in its relatively large shape (9). The fundamental factor for the enlargement of the VA is enlargement of the endolymphatic sac and endolymphatic vessels. The endolymphatic fluid produced by the cochlea flows through the endolymphatic vessels and endolymphatic sac and transmits pressure in the membranous labyrinth. Under normal development, the narrow VA and membranous labyrinth inside the cochlear aqueduct protect the inner ear against rapidly rising intracranial pressure. However, even mild traumatic brain injury can cause fluctuations in cerebrospinal fluid pressure when the anterior atrium aqueduct is enlarged and the cochlear aqueduct is normal. Conduction to the inner ear by the significantly enlarged endolymphatic vessels and endolymphatic sac ruptures the membranous labyrinth, causing mixing of internal and external lymphatic fluid, presenting as sudden deafness and vertigo (10-13). This sudden onset of deafness can recover by itself or after treatment, but repeated attacks can lead to total deafness. This is the basic progression of LVAD deafness. Up till now, there has not been any other reversible method than artificial hearing aid technology.
With the development of science and technology and social progress, Chinese enterprises had begun to produce cochlear implant system, an active implantable medical device that had been implanted into human body for a long time. Due to the high installation and use risks of this kind of products, there is no corresponding national or industry standard or international standard for transformation and demonstration in China at present, and the evidence of safety and effectiveness submitted by various studies was quite different from the existing technical evaluation requirements. Cochlear implant is based on the following considerations for the reconstruction of hearing in the large vestibule: the main reason is that the incidence of the large vestibule is mostly after the language period, and even has a certain language foundation. Under the condition of ensuring the normal development of the auditory nerve, the effect of cochlear implant is guaranteed (14). A cochlear implant is better than a hearing aid for LVAD patients whose hearing loss has reached the more than severe level.

Because anatomic abnormality of the inner ear structure will increase the risk of cochlear surgery, a certain number of neurons must be equipped to effectively transmit signals to the auditory cortex. Therefore, the safety and effectiveness of cochlear implants in these patients are controversial. The innovation of this study is our metaanalysis to compare the categories of auditory performance, speech intelligibility rate and duration of cochlea use of cochlear implant in deaf patients with inner ear malformations and those with normal anatomic structure of the inner ear. We explored whether cochlear implantation can improve hearing in patients with LVAD to provide reliable evidence-based evidence for clinical treatment.

We present the following article in accordance with the PRISMA reporting checklist (available at https://dx.doi. org/10.21037/apm-21-3327).

\section{Methods}

\section{Document retrieval}

A literature search was carried out in the CNKI, Baidu Academic, Weipu Medical, Wanfang Data, PubMed, EBSCO, Medline, and Cochrane databases. Randomized controlled trials (RCTs) of cochlear implants for the treatment of LVAD published from the establishment of the database to July 20, 2021 were retrieved. English search terms included: Large vestibular canal malformation, Deafness, large vestibular aqueduct, and Cochlear implants. 
Chinese search terms included: Large vestibular aqueduct deformity (LVAD), Deafness, and Cochlear implant. A combination of subject terms and free words was applied to perform multiple searches to obtain reference documents that could be included, and then a search engine was used to track each document. Eventually, relevant experts and researchers who had published in the literature were contacted to obtain the latest research progress. The quality of the literature was evaluated by RevMan 5.3 provided by the Cochrane Collaboration.

\section{Literature inclusion and exclusion criteria}

Inclusion criteria were: (I) RCT design method; (II) imaging results showed only deformity of the large vestibular aqueduct; (III) subjects had sensorineural hearing loss; (IV) cochlear implant or follow-up for $>1$ year; (V) normal cognitive function of the patient before surgery.

Exclusion criteria were: (I) individual cases or nonresearch literature; (II) not a RCT; (III) duplicate literature, unknown data and information or no original data.

\section{Measurement indexes}

Auditory skill, Categories of auditory performance (CAP), speech intelligibility rate (SIR), Word recognition score, Pure tone audiometry, Duration of cochlea use, Behavioral threshold, intracochlear resistance, speech detection thresholds, CNC monosyllabic word test, CUNY sentence test, Categories of auditory performance, speech intelligibility rate.

\section{Data extraction}

Two researchers used unified Microsoft Excel (Microsoft, USA) to independently conduct literature screening and data extraction, and then cross-checked them. If there were differences, they were resolved through discussion. The main extracted data included: (I) baseline bibliographic information such as title, name of the first author, published journal, and publication year; (II) baseline clinical information such as sex, age, and number of cases; (III) specific intervention measures and follow-up time; (IV) extraction of determination indexes.

\section{Risk of bias assessment}

The included RCTs were simultaneously assessed for bias risk by two researchers. Differences were resolved through discussion. In this study, the JADAD rating scale was used to evaluate literature quality. The evaluation factors included random sequence generation, allocation hiding, and blind method. The contents of the included literature were scored according to the JADAD scale, ranging from 0 to 6 . If the number of patients included in the literature was large, the degree of matching was high, the more evaluation indexes, the higher the score, and the higher the quality of the included literature.

\section{Statistical methods}

Stata SE12.0 was used for statistical analysis, with the odds ratio (OR) used for binary variables, and mean difference (MD) for continuous variables. The RevMan 5.3 bias risk assessment chart was used to assess the risk bias of the included references, and the data were sorted, filtered, and then input into RevMan 5.3 to draw the chart. Each effect was represented by a $95 \%$ confidence interval (CI). When $\mathrm{P}>0.01$ and $\mathrm{I}^{2}<50 \%$, the fixed-effects model was used for meta-analysis, but when $\mathrm{P}<0.01$ and $\mathrm{I}^{2}>50 \%$, the randomeffects model was used.

\section{Sensitivity analysis}

Random-effects and fixed-effects models were used to compare the results. According to their consistency, the reliability of the combined results was analyzed, and a funnel chart was used to determine whether there was publication bias.

\section{Results}

\section{Search results and basic document information}

A total of 185 documents were obtained by searching the database and 68 documents were obtained by searching the register; 53 duplicate publications, 35 unqualified studies and 16 rejected for other reasons were eliminated, leaving 149 papers. After 48 and 76 papers, respectively, were eliminated, 25 papers remained, of which 20 were excluded, comprising 13 reviews, 3 incomplete studies, and 4 not about LVAD, leaving 5 to be included in the metaanalysis. Figure 1 is the flow chart of literature retrieval and screening.

The 5 articles included 567 patient cases. All were small-sample studies, with sample size ranging from 12 to 


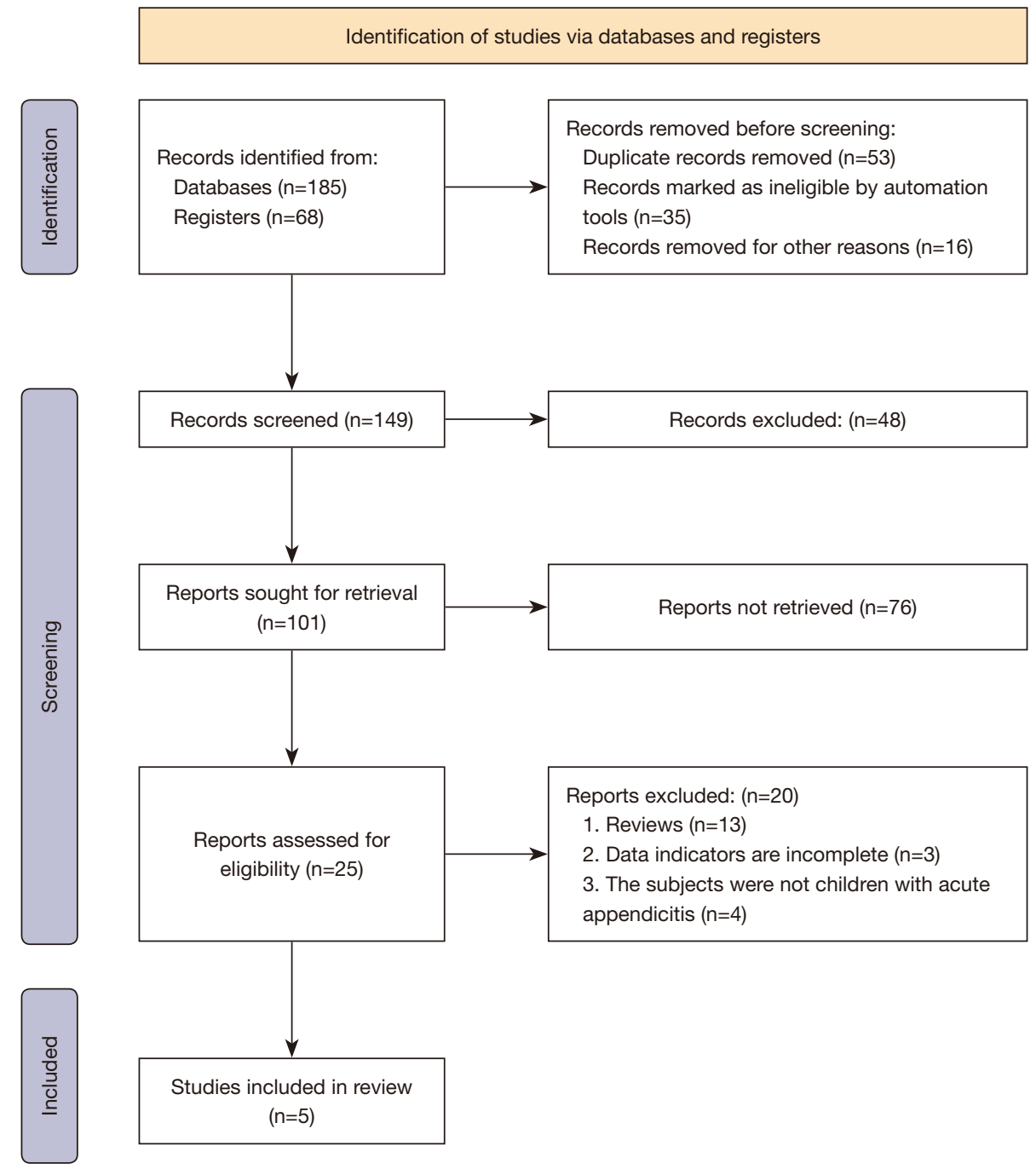

Figure 1 Literature screening process.

435. The 5 articles had detailed descriptions of CAP, SIR, Duration of cochlea use in a deformity group and a control group. Table 1 shows the baseline characteristics of the included literature.

\section{Results of risk bias evaluation of the literature}

Figures 2,3 are the risk bias evaluation diagram and summary diagram, respectively, drawn by RevMan 5.3. Of the 13 RCTs, two $(15,16)$ described the correct random allocation method, accounting for $40 \%$. Only one article (17) described concealment of the allocation plan in detail, accounting for $20 \%$. The remaining $(18,19)$ did not use blinding, accounting for $40 \%$.

\section{Comparison of categories of auditory performance}

There were 2 RCTs that analyzed hearing ability and Figure 4 is a forest plot of the random-effects model. In 2 studies, dichotomous variables were used to describe hearing ability in a total of 48 cases, including 24 cases in the deformity group and 24 cases in the control group. For the heterogeneity test of the whole population, $\mathrm{Chi}^{2}=4.00$, $\mathrm{df}=1, \mathrm{I}^{2}=75 \%>50 \%$, and $\mathrm{P}=0.05$. Overall analysis using a random-effects model showed no statistically considerable difference between the deformity group and the control group ( $\mathrm{Z}=0.78, \mathrm{MD}=-0.65,95 \% \mathrm{CI}:-2.29$ to $0.98, \mathrm{P}=0.43$ ). There was no substantial difference in postoperative hearing ability between LVAD patients and those with normal inner ear structure. 
Table 1 Baseline information of the included studies

\begin{tabular}{lccccl}
\hline Author & Year & Patients (n) & Deformity group (n) & Normal group (n) & Outcome indicators \\
\hline $\begin{array}{l}\text { Chen (15) } \\
\text { Demir (16) }\end{array}$ & 2011 & 435 & 62 & 373 & Auditory skill \\
& 2020 & 36 & 18 & 18 & $\begin{array}{l}\text { Categories of auditory performance, speech intelligibility } \\
\text { rate, word recognition score, pure tone audiometry, } \\
\text { duration of Cl use }\end{array}$ \\
Jahn (17) & 2020 & 15 & 5 & 10 & Behavioral threshold, intracochlear resistance \\
Miyamoto (18) & 2002 & 69 & 23 & 46 & $\begin{array}{l}\text { Mean length of Cl use, speech detection thresholds, CNC } \\
\text { monosyllabic word test, CUNY sentence test }\end{array}$ \\
& 2012 & 12 & 6 & 6 & $\begin{array}{l}\text { Categories of auditory performance, speech intelligibility } \\
\text { rate }\end{array}$ \\
\hline
\end{tabular}

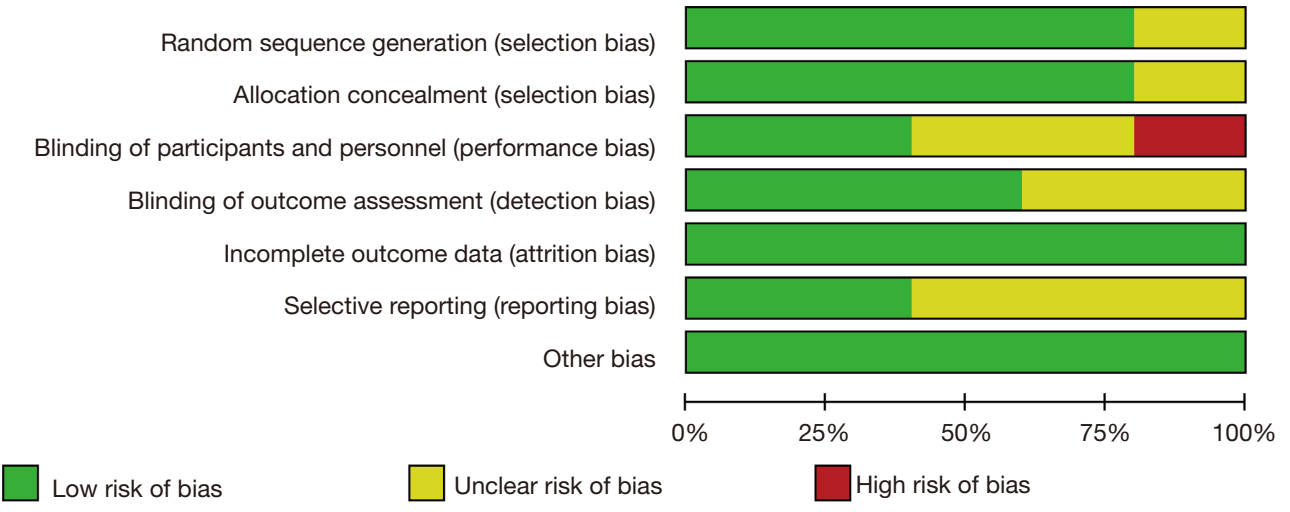

Figure 2 Evaluation of the risk bias of the included literature.

Figure 5 is the funnel plot of categories of auditory performance. The circles and midlines included in the study are basically symmetrical, suggesting that the study had high precision, there was no publication bias, and the final conclusion was relatively credible.

\section{Comparison of speech intelligibility rate}

The speech intelligibility rate was analyzed in RCTs and Figure 6 is the forest plot of the fixed-effects model. Continuous variables were used to describe the SIR in a total of 48 cases, including 24 cases in the deformity group and 24 cases in the control group. For the heterogeneity test of the whole population, $\mathrm{Chi}^{2}=1.75, \mathrm{df}=1, \mathrm{I}^{2}=43 \%<50 \%$, and $\mathrm{P}=0.19$. Overall analysis using a fixed-effects model showed that the difference between the deformity group and the control group was statistically considerable $(\mathrm{Z}=3.09$, $\mathrm{MD}=-1.03,95 \% \mathrm{CI}:-1.69$ to $-0.38, \mathrm{P}=0.002)$. The results indicated there was no substantial difference between LVAD patients and patients with normal inner ear structure.

Figure 7 is the funnel plot of speech intelligibility rate. The circles and midlines included in the study are basically symmetrical, suggesting that the study had high precision, there was no publication bias, and the final conclusion was relatively credible.

\section{Comparison of duration of cocblea use}

Two RCTs analyzed the postoperative complications and Figure 8 is a forest plot of the random-effects model. There were a total of 105 cases, including 41 cases in the deformity group and 64 cases in the control group. For the heterogeneity test of the whole population, $\mathrm{Chi}^{2}=2.14$, $\mathrm{df}=1, \mathrm{I}^{2}=53 \%>50 \%$, and $\mathrm{P}=0.14$. Overall analysis using a random-effects model shows no statistically considerable difference between the deformity group and the control group ( $\mathrm{Z}=1.17, \mathrm{MD}=-1.81,95 \% \mathrm{CI}:-4.86$ to $1.23, \mathrm{P}=0.24)$, The results indicated there was no substantial difference 
between LVAD patients and patients with normal inner ear structure.

Figure 9 is the funnel plot of duration of cochlea use. The circles and midlines included in the study are basically symmetrical, suggesting that the study had high precision, there was no publication bias, and the final conclusion was relatively credible.

\section{Discussion}

LVAD is a common cause of severe sensorineural hearing loss. A few cases are mixed or conductive hearing loss in the early stage. Most show pre-language deafness, and

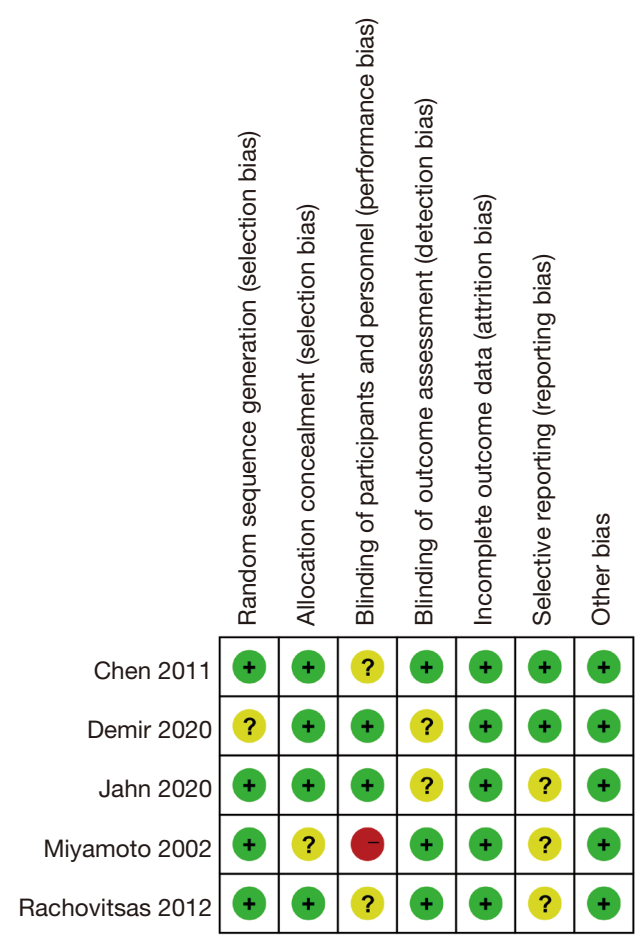

Figure 3 Summary diagram of risk bias evaluation of the included literature. some show post-language deafness. Studies have found that the vast majority of patients with LVAD-induced postspeech hearing loss develop hearing loss of 3-4 dB/year, eventually progressing to severe to extremely severe sensorineural hearing loss (20-22). Compared with patients with normal inner ear structure, the probability of facial nerve abnormality after LVAD implantation in cochlea was higher. The facial nerve abnormalities were mostly anterior displacement, which caused the facial nerve recess to narrow and thus affects the exposure of the round window niche. It was necessary for the operator to locate the facial nerve according to the relatively fixed anatomical landmarks such as the outer semicircular canal, incus fossa, spoon process and cone eminence, and actively and thoroughly contour the facial nerve and chorda tympani nerve, so as to increase the width of the facial nerve recess and obtain a good surgical vision, thereby reducing the risk of facial nerve injury and the occurrence of postoperative complications $(23,24)$. In this study, the efficacy of cochlear implant was compared between LVAD patients and deaf patients with normal inner ear structure. We found that a few patients in both groups had blowouts, and lymph fluctuation was clearly observed when the cochlea was drilled. After electrode implantation, the round window can be filled with temporalis fascia and muscle. The literature on blowouts suggests that the reason for blowout is that the patients' vestibular and inner auditory canal floors have different degrees of communication, and a high degree of deformity is related to whether the vestibular aqueduct is open (25). Some patients may have postoperative complications such as vertigo, nausea, and vomiting. If timely symptomatic anti-inflammatory treatment is given for two days, no infection occurs. There was no substantial difference in the electrode impedance between LVAD patients and patients with normal inner ear structure in this analysis, which may be related to the tissue environment around the electrode and the performance of the product itself.

The hearing ability and SIR of the patients in the

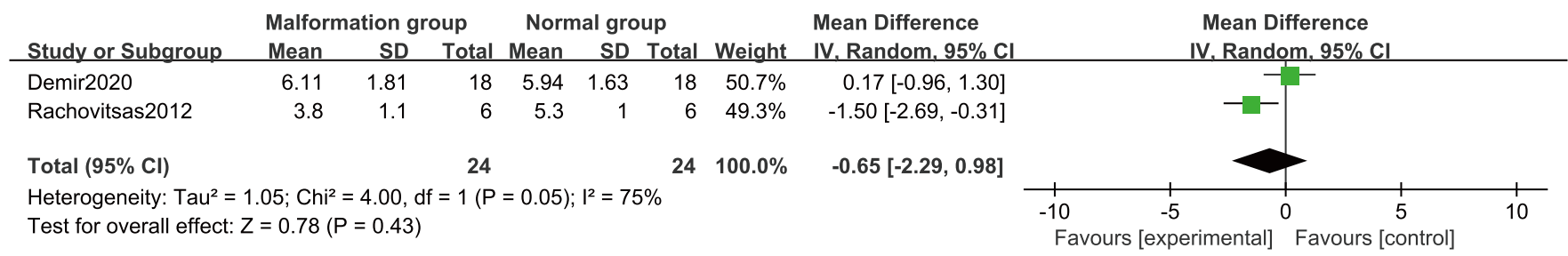

Figure 4 Forest plot of random-effects model of categories of auditory performance. 


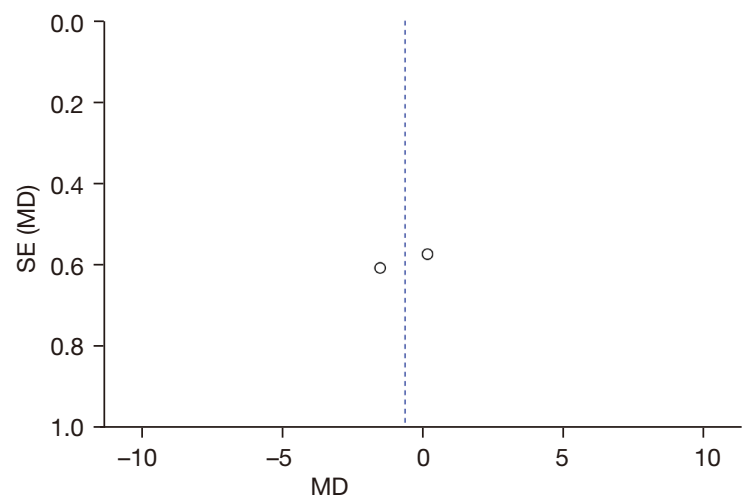

Figure 5 Funnel chart of categories of auditory performance. SE, standard error; $\mathrm{MD}$, mean difference.

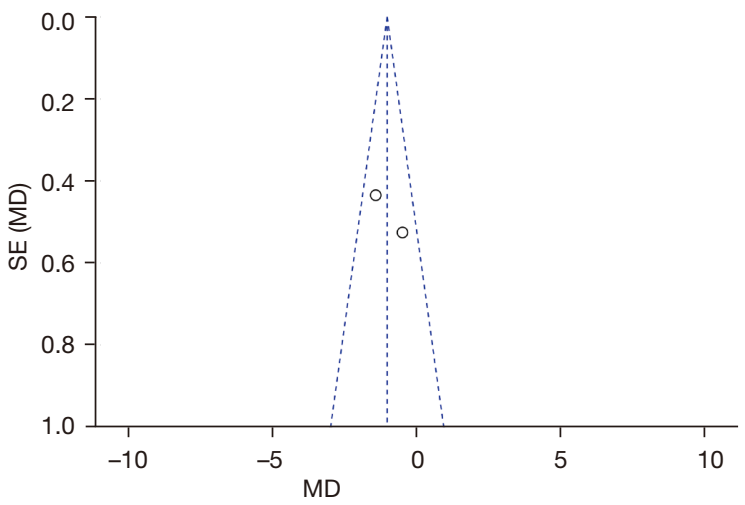

Figure 7 Funnel chart of speech intelligibility rate. SE, standard error; MD, mean difference.

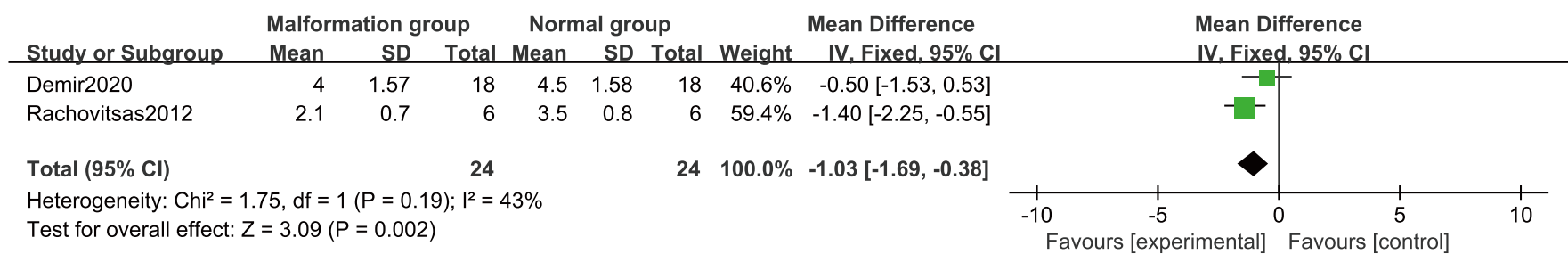

Figure 6 Forest plot of fixed-effects model of speech intelligibility rate.

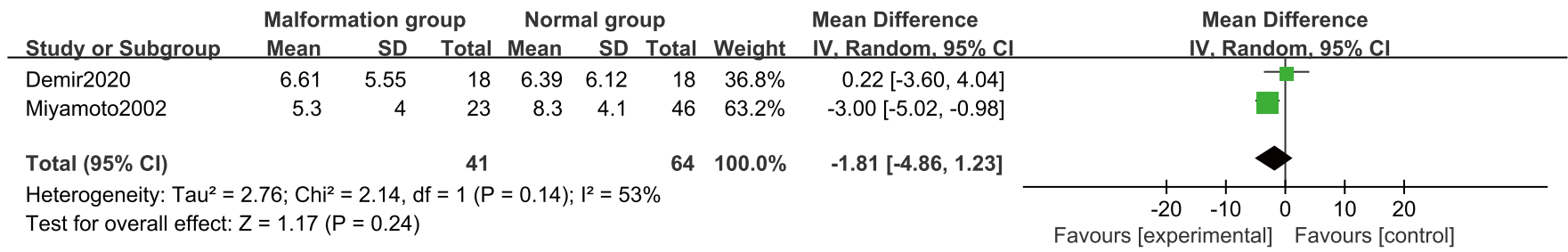

Figure 8 Forest plot of random-effects model of duration of cochlea use.

two groups were analyzed and we found no substantial difference between patients with LVAD and deaf patients with normal inner ear structure, which indicates that the cochlear implant can be applied to LVAD patients with substantial clinical effect. According to Ehrmann-Müller et al. in 2020 (26), only 4,500 ganglion cells are needed to maintain SIR in cochlear implant patients. Moreover, the clinical efficacy of cochlear implants for LVAD patients is better than hearing aids. Before cochlear implant, the MRI and CT images of patients should be carefully examined. Intraoperative monitoring methods such as facial nerve monitor, electrically stimulated auditory evoked potential, and nerve response telemetry can significantly reduce the incidence of complications $(27,28)$. Learning speech and communication require rehabilitation training after surgery and can significantly promote the recovery of patients' hearing ability.

The JADAD score results showed that $40 \%$ of the literature had scores of 3-6, indicating sufficiently advanced quality of the studies, and the included experiments had good quality and high reliability. According to the funnel plot of listening ability, the circles and the middle line were basically symmetrical, suggesting high precision of the study, no publication bias, and a relatively credible final conclusion. 


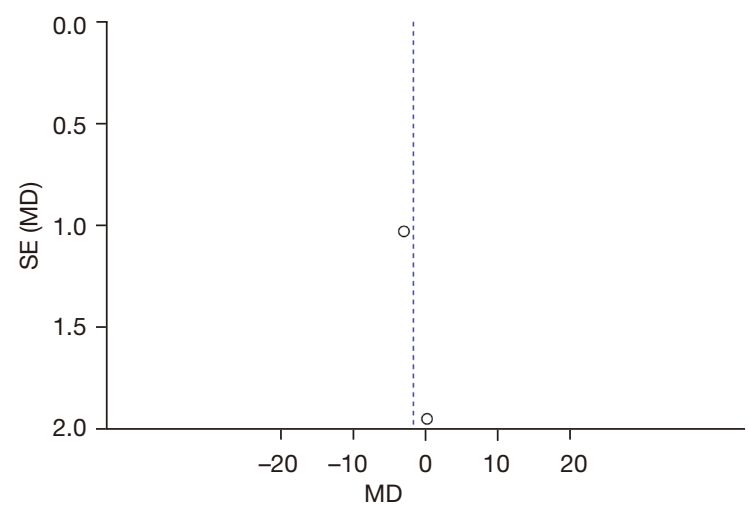

Figure 9 Funnel chart of duration of cochlea use. SE, standard error; $\mathrm{MD}$, mean difference.

\section{Conclusions}

In this study, LVAD patients with cochlear implant were set as the deformity group and patients with normal inner ear structure as the normal group for the meta-analysis exploring the efficacy and safety of cochlear implant in patients with LVAD. The results confirmed that with postoperative rehabilitation of LVAD patients with cochlear implantation the clinical efficacy is similar to that of patients with normal inner ear structure, providing an important basis for hearing restoration. However, this study also has some limitations. Some of the included literature did not have all the measurement indicators, and only a few papers mentioned individual indicators. In the future, more comprehensive indicators should be used for comprehensive analysis. In conclusion, cochlear implantation has a positive effect in LVAD patients, and with postoperative rehabilitation the effect is similar to that of deaf patients with normal inner ear structure. In the future, a greater sample size is needed to verify the effectiveness of cochlear implantation in patients with LVAD.

\section{Acknowledgments}

Funding: None.

\section{Footnote}

Reporting Checklist: The authors have completed the PRISMA reporting checklist. Available at https://dx.doi. org/10.21037/apm-21-3327

Conflicts of Interest: All authors have completed the ICMJE uniform disclosure form (available at https://dx.doi. org/10.21037/apm-21-3327). The authors have no conflicts of interest to declare.

Ethical Statement: The authors are accountable for all aspects of the work in ensuring that questions related to the accuracy or integrity of any part of the work are appropriately investigated and resolved.

Open Access Statement: This is an Open Access article distributed in accordance with the Creative Commons Attribution-NonCommercial-NoDerivs 4.0 International License (CC BY-NC-ND 4.0), which permits the noncommercial replication and distribution of the article with the strict proviso that no changes or edits are made and the original work is properly cited (including links to both the formal publication through the relevant DOI and the license). See: https://creativecommons.org/licenses/by-nc-nd/4.0/.

\section{References}

1. Yan YJ, Li Y, Yang T, et al. The effect of GJB2 and SLC26A4 gene mutations on rehabilitative outcomes in pediatric cochlear implant patients. Eur Arch Otorhinolaryngol 2013;270:2865-70.

2. Bichey BG, Hoversland JM, Wynne MK, et al. Changes in quality of life and the cost-utility associated with cochlear implantation in patients with large vestibular aqueduct syndrome. Otol Neurotol 2002;23:323-7.

3. Clarós P, Fokouo JV, Clarós A. Cochlear implantation in patients with enlarged vestibular aqueduct. A case series with literature review. Cochlear Implants Int 2017;18:125-9.

4. Harker LA, Vanderheiden S, Veazey D, et al. Multichannel cochlear implantation in children with large vestibular aqueduct syndrome. Ann Otol Rhinol Laryngol Suppl 1999;177:39-43.

5. Li Y, Yang Y, Zhang W, et al. Developmental performance between pediatric cochlear implantation candidates with and without large vestibular aqueduct syndrome. Acta Otolaryngol 2021;141:250-5.

6. Mey K, Bille M, Cayé-Thomasen P. Cochlear implantation in Pendred syndrome and non-syndromic enlarged vestibular aqueduct - clinical challenges, surgical results, and complications. Acta Otolaryngol 2016;136:1064-8.

7. Powell HR, Birman CS. Large vestibular aqueduct syndrome: Impedance changes over time with different cochlear implant electrode arrays. Cochlear Implants Int 
2015;16:326-30.

8. Au G, Gibson W. Cochlear implantation in children with large vestibular aqueduct syndrome. Am J Otol 1999;20:183-6.

9. Vassoler TM, Bergonse Gda F, Meira Junior S, et al. Cochlear implant and large vestibular aqueduct syndrome in children. Braz J Otorhinolaryngol 2008;74:260-4.

10. Kontorinis G, Goetz F, Giourgas A, et al. Radiological diagnosis of incomplete partition type I versus type II: significance for cochlear implantation. Eur Radiol 2012;22:525-32.

11. Asma A, Anouk H, Luc VH, et al. Therapeutic approach in managing patients with large vestibular aqueduct syndrome (LVAS). Int J Pediatr Otorhinolaryngol 2010;74:474-81.

12. Chen YW, Wu CM. Cochlear implantation in patients with large vestibular aqueduct syndrome (LVAS). Cochlear Implants Int 2004;5 Suppl 1:124-6.

13. Clark JL, Roeser RJ. Large vestibular aqueduct syndrome: a case study. J Am Acad Audiol 2005;16:822-8.

14. Vashist S, Singh S. CSF Gusher in Cochlear Implant Surgery-does it affect surgical outcomes? Eur Ann Otorhinolaryngol Head Neck Dis 2016;133 Suppl 1:S21-4.

15. Chen X, Liu B, Liu S, et al. The development of auditory skills in infants with isolated Large Vestibular Aqueduct Syndrome after cochlear implantation. Int J Pediatr Otorhinolaryngol 2011;75:943-7.

16. Demir B, Cesur S, Incaz S, et al. The effect of canal diameter on audiologic results in patients with cochlear implantation with large vestibular aqueduct syndrome. Eur Arch Otorhinolaryngol 2020;277:743-50.

17. Jahn KN, Bergan MD, Arenberg JG. Auditory Detection Thresholds and Cochlear Resistivity Differ Between Pediatric Cochlear Implant Listeners With Enlarged Vestibular Aqueduct and Those With Connexin-26 Mutations. Am J Audiol 2020;29:23-34.

18. Miyamoto RT, Bichey BG, Wynne MK, et al. Cochlear implantation with large vestibular aqueduct syndrome. Laryngoscope 2002;112:1178-82.

19. Rachovitsas D, Psillas G, Chatzigiannakidou V, et al.

Cite this article as: Pan L, Lin H, Li X, Liu S. Systematic review and meta-analysis of cochlear implantation in deaf patients with large vestibular aqueduct deformity. Ann Palliat Med 2021;10(12):12598-12606. doi: 10.21037/apm-21-3327
Speech perception and production in children with inner ear malformations after cochlear implantation. Int J Pediatr Otorhinolaryngol 2012;76:1370-4.

20. Yang HL, Liu Z. The effect of optimizing EABR parameters in artificial cochlear implantation for auditory rehabilitation. Eur Rev Med Pharmacol Sci 2017;21:2015-20.

21. Almaramhy HH. Acute appendicitis in young children less than 5 years: review article. Ital J Pediatr 2017;43:15.

22. Hall AC, Kenway B, Sanli H, et al. Cochlear Implant Outcomes in Large Vestibular Aqueduct Syndrome-Should We Provide Cochlear Implants Earlier? Otol Neurotol 2019;40:e769-73.

23. Hura N, Stewart M, Walsh J. Progression of hearing loss and cochlear implantation in large vestibular aqueduct syndrome. Int J Pediatr Otorhinolaryngol 2020;135:110133.

24. Sullivan JC, Johnson CE, Danhauer JL, et al. Large Vestibular Aqueduct Syndrome: Parents' and Audiologists' Perspectives. J Am Acad Audiol 2019;30:677-93.

25. Tolisano AM, Schauwecker N, Baumgart B, et al. Identifying Disadvantaged Groups for Cochlear Implantation: Demographics from a Large Cochlear Implant Program. Ann Otol Rhinol Laryngol 2020;129:347-54.

26. Ehrmann-Müller D, Shehata-Dieler W, Kaulitz S, et al. Cochlear implantation in children without preoperative computed tomography diagnostics. Analysis of procedure and rate of complications. Int J Pediatr Otorhinolaryngol 2020;138:110266.

27. Lee Y, Sim H. Bilateral cochlear implantation versus unilateral cochlear implantation in deaf children: Effects of sentence context and listening conditions on recognition of spoken words in sentences. Int J Pediatr Otorhinolaryngol 2020;137:110237.

28. Kim SC, Oh YL, Lee HS. Relation between CSF gusher and large endolymphatic sac in the enlarged vestibular aqueduct syndrome during cochlear implantation. Cochlear Implants Int 2004;5 Suppl 1:78-9. 